Research Article

\title{
The Construct Validity of Vallerand's Academic Motivation Scale (AMS)
}

\author{
Salem Ali Salem Algharaibeh \\ Department of Educational Sciences, Ajloun University College, Al-Balqa Applied University, Salt, Jordan \\ Correspondence should be addressed to Salem Ali Salem Algharaibeh; salem.algharibeh@bau.edu.jo
}

Received 13 February 2021; Revised 24 March 2021; Accepted 27 March 2021; Published 7 April 2021

Academic Editor: Haoran Xie

Copyright (c) 2021 Salem Ali Salem Algharaibeh. This is an open access article distributed under the Creative Commons Attribution License, which permits unrestricted use, distribution, and reproduction in any medium, provided the original work is properly cited.

\begin{abstract}
It seems that the academic motivation structure is affected by cultural factors. Many studies have examined the factorial structure of the academic motivation scale (AMS), and the results showed different factorial structures of AMS (e.g., Taghipour Ali Hosein et al. (EL-yazidi and Louzani, 2017) concluded that the scale consists of two dimensions; Natalya and Purwanto (2018) concluded that it consists of three dimensions; Alruaili (2020) concluded that it consists of four dimensions; Abu Awad (2009) concluded that the scale consists of six dimensions). The AMS is one of the most widely used academic motivation measures across the world. It was built on the basis of the self-determination theory. The current study aimed at investigating the factorial structure of the AMS using the exploratory factor analysis (PCA) and the confirmatory factor analysis (CFA). The AMS was applied to a sample of 401 university students. The results of PCA suggested a three-factor solution (intrinsic motivation, extrinsic motivation, and Amotivation), and CFA was conducted for three competing structures (three factors, five factors, and seven factors); the results confirmed the three-factor solution for the AMS. The results also showed that the AMS dimensions had good alpha coefficient values which were greater than the acceptable cut-off value of 0.7. In conclusion, the Jordanian version of the AMS is a valid scale that consists of 24 items loaded on three factors (intrinsic, extrinsic, and Amotivation) for measuring academic motivation.
\end{abstract}

\section{Introduction}

Motivation is a state of tension that activates living organisms to carry out certain patterns of behaviour, which lead to the satisfaction of the motive or at least the reduction of its strength so that the balance is achieved in the organism. This balance is the rest as a result of reducing tension [1].

Atkinson [2] defined motivation as the current subjective factors affecting an individual's direction, strength, and persistence in action [3]. Schunk [4] defined it as the process of inducing and maintaining behaviour. It indicates arousal, orientation, and perseverance in behaviour [5]. It may also be seen as a power that causes people to engage in certain behaviour [6].

Motivation complexity not only means that different people have different motivations to do the same thing, but also that the same person may have different motives for the same behaviour at different times [7]. As a result of the complexity of the concept of academic motivation and its central role in the educational field, many studies have attempted to capture most aspects of academic motivation within the framework of the self-determination theory, which determines that human behaviour is formed through the interaction between an individual and his/her social environment and affects the motivation of individuals due to the psychological and natural needs that they try to satisfy, regardless of the social environment they belong to [8] Many applications of this theory have been found in several fields, including the educational and academic fields [9].

Motivation has two sides, one of them is internal and the other is external, which make motivation a cause and a directive for behaviour and not just an internal push without a goal [10]. Haider was one of the first to distinguish between intrinsic and extrinsic motivation. Accordingly, behaviour that occurs as a result of internal motives is considered to be under the control of intentional behaviour, while behaviour 
that results from external motivation is unintentional behaviour that is under the control of external factors [11].

Motivational orientation refers to the motivational attitude that the student adopts in learning inside the classroom, and thus the student integrates it into school activities for internal or external reasons [12]. Students' motivational attitudes and beliefs about the learning process are related to cognitive engagement and classroom performance [13]. So far, motivation research in the classroom has focused on the existence of these internal/external trends and the various influences resulting from them [14].

Intrinsic motivation represents the desire to perform the task for its own sake, while extrinsic motivation represents the desire to perform the task to obtain external rewards or avoid punishment [15]. The intrinsic motivation corresponds to the desire to learn, while extrinsic motivation corresponds to the need to learn, both of which lead to successful learning [16]. Additionally, intrinsic motivation is motivated by enjoyment (enjoyment-driven), while external motivation is driven by reinforcement (reinforcementdriven) [17].

The concepts of intrinsic and extrinsic motivation are not simple, as they include different types of students' motivation and attitudes. They perceive intrinsic motivation as being provoked by the task itself (learning to learn), in addition to the student's internal needs (curiosity and self-realisation), whereas extrinsic motivation is usually perceived as being provoked by extrinsic rewards [18].

Internal motivation and external motivation are not contradictory. Deci and Ryan [19] argue that the basis for this is the extent to which individuals perceive their behaviour as self-determined. Internally driven behaviours are self-determined, while externally driven behaviours are not self-determined. If individuals find behaviours consistent with their self-image and consistent with their preferences and values, then they will perceive themselves as self-determiners of their decisions even if they receive external rewards, but if they realise that their actions are not consistent with their preferences and desires, i.e., they are done to obtain external rewards, then the rewards will reduce motivation [20].

Among the manifestations of intrinsic motivation are feeling happy in learning, orientation towards learning, integration into task [21], increasing effort, feeling of adequacy, interest, low level of anxiety and nervousness [22], curiosity, learning difficult tasks and challenging tasks, perseverance, orientation of mastery, a high level of engagement in the task [23], control over results, a feeling of congruence between effort and outcome [24], control over the learning situation [11], and high academic achievement [25].

Researchers differ as to whether there are general motives for all cultures or that they differ from one individual to another. Allport [26] mentioned that there are no common motives to all human beings, for every individual is a fabric alone, just as every civilisation is a fabric alone, while McClelland suggested that there is an existence of general motives in which all human beings share [27].
Goals pursued by any educational system and the importance of motivation from the educational point of view are evident in that it is a method that can be used to effectively reach specific educational goals by considering it as one of the determining factors for the student's ability to achieve academic achievement [28].

In the educational and academic field, the self-determination theory (SDT) assumes a multidimensional perspective of academic motivation, which can be arranged on a continuum of self-determination. At the upper end of the continuum, we find intrinsic motivation, and then we find extrinsic motivation, and at the end of the continuum of selfdetermination we find Amotivation.

Motivation to learn is a distinct case of general motivation. It is clear that stimulating the general motivation to learn is a huge task that the school alone is not sufficient to do; it is a task shared by the school, home, community institutions, and culture in general [27]. Deci and Ryan [29] argue that stimulating students' academic motivation makes their learning more effective and their school and class interaction more positive and increases their enthusiasm to participate in classroom learning situations. Academic motivation is important in increasing the student's attention and their time engagement into educational activities, focusing their attributions in success and failure to internal factors. This helps them in controlling the factors affecting the achievement of the learning mission, all of which contribute to increasing their effort, controlling their learning experiences, and increasing their motivation. McClelland [30] was interested in studying academic motivation, followed by [31]; all of whom concluded that success and failure are related to students' level of motivation to learn.

Through this complex presentation of the concept of academic motivation, there is a need to develop tools with acceptable psychometric properties of validity and reliability. Several studies have been concerned with designing measures of motivation, such as the Academic Internal Motivation Scale [32], Academic Intrinsic and Extrinsic Motivation Scale [33], and Academic Motivation Scale (AMS) [34].

Vallerand et al. [34] developed the AMS, which is based on theoretical frame of self-determination theory [19]. The AMS is one of the most popular measures of motivation in the academic area [35]. The AMS that was composed by Vallerand et al. [34] in its French version (original version) or the English version has been searched for in many countries over the world to verify the factorial structure of the scale.

Vallerand et al. [34] applied the confirmatory factor analysis of the seven-scale factors. They extracted Cronbach's alpha coefficient of the scale and the reliability of the scale was also verified by the test-retest method. The results suggested that the seven-factor model had the best fit.

Vallerand et al. [34] studied the psychometric properties of the scale using construct validity, by finding correlations between the scale dimensions and other measures of motivation. They found the correlations that supported the relationships between all dimensions of the scale and other 
measures, but the intrinsic motivation to experience stimulation (IMES) showed weak correlations with some other measures of academic motivation. This means that there is a lack of confidence in the factorial structure of this dimension specifically.

Cokley [36] examined the psychometric properties of the AMS on an American sample, and the results concluded that the differences between internal and external motivation are unclear. Also, the correlation between Amotivation with intrinsic motivation subscales was negative and not statistically significant. In another study conducted by [37], examining the factorial structure of the AMS on another sample of American students, the results supported the seven-factor model of the scale.

Fairchild et al. [38] studied the validity of AMS fitness indicators, and their findings concluded the fitness of the seven-factor model and extracted the indications of the convergent and discriminatory validity of the scale. However, they indicated that the scale factors need further investigation in their current form before it is finally adopted. EL-yazidi and Louzani [39] checked the factorial structure of AMS using an exploratory factor analysis method, and the results showed two factors: intrinsic and extrinsic motivation. Stover et al.'s [35] results also supported the sevenfactor model of the scale using the confirmatory factor analysis on an Argentine sample.

The AMS has already been used and tested in many countries. Cokley et al. [37] conducted a validation of the AMS in the United States. Ochoco [40] validated the AMS in the Philippines. Alivernini and Lucidi [41] validated the AMS in Italy and Karaguven [42] in Turkey. Lim and Chapman [43] also conducted the validation of the AMS in Singapore. Caleon et al. [44] conducted a cross-cultural validation in Singapore; Zhang et al. [45] conducted a validation of AMS in China. Natalya and Purwanto [46] conducted a validation of AMS in Indonesia. The factorial structure of the AMS was also verified in the Arab environment by $\mathrm{Abu}$ Awad [47], who aimed to examine the factorial structure of the academic motivation scale on a sample of sixth- and tenth-grade students in the United Nations Relief and Works Agency for Palestine Refugees in the Near East (UNRWA) schools in Jordan. The results did not support the seven-factor model for the scale as the results of the factor analysis showed six factors of academic motivation. The current study aimed to apply confirmatory factor analysis (CFA) and exploratory factor analysis (EFA) to examine the validity of the AMS in Jordan.

All these previous studies agreed that the AMS is suitable for measuring academic motivation, but they differed with regard to its factorial structure and the number of factors that compose it. To ensure accurate measurement of academic motivation in Jordan, the validation of the AMS is, therefore, of great importance.

\section{Methods}

2.1. Participants. The population of this study involved all public university students in Jordan (210976 students). The sample consisted of 401 students who were selected randomly (simple random-online questionnaires) from the study population. $283(70.6 \%)$ participants were female and $118(29.4 \%)$ were male. The participants responded to the AMS in the summer semester of the 2019-2020 academic year.

2.2. Academic Motivation Scale (AMS). The theoretical framework for the AMS depends on the self-determination theory of Deci and Ryan $[19,48]$, which assumes the existence of multiple factors of academic motivation, arranged along the continuum of the self-determination as follows: intrinsic motivation, extrinsic motivation, and Amotivation.

Vallerand et al. [34] suggested three factors for intrinsic motivation: the intrinsic motivation to know (IMTK) which means that the student performs the activity for the pleasure that they feel when they got a new learning; the intrinsic motivation to accomplish (IMTA) which means that the student interacts with the environment to feel competent; and Intrinsic Motivation to Experience Stimulation (IMES) which means the student engages in the assignment to experience stimulating. The three factors of intrinsic motivation exist in the continuum of self-determination, but they are factors of intrinsic motivation with a correlation between each other. Three factors for the extrinsic motivation are extrinsic motivation for external regulation (EMER) which means that the student performs the activity in order to get external reinforcement, extrinsic motivation for introjected regulation (EMIN) which means that the student begins to personalize his actions reasons, extrinsic motivation for identified regulation (EMID) which makes the behaviour valuable and important for the student, and one factor for the Amotivation (AMOT) which means that the student does not have intrinsic or extrinsic motivation. Each subscale consists of four items each.

The AMS consists of 28 items measuring seven factors. Vallerand et al. [34] investigated its reliability using Cronbach's alpha coefficient, which ranged between 0.83 and 0.86; they also investigated the convergent validity of the scale, by examining the relationship between the scale's dimensions and other measures of motivation. The results confirmed the validity of the AMS as a measure of motivation. Algharaibeh [49] investigated the psychometric properties of the AMS in the Jordanian environment, and the results showed that the AMS has an acceptable validity and reliability.

2.3. Research Procedure. The AMS [34] was administrated to the study sample (online) in the summer semester of the 2019-2020 academic year.

\section{Results}

3.1. Reliability. The reliability measures were assessed using Cronbach's alpha coefficient as shown in Table 1.

Table 1 shows that the AMS dimensions had good alpha coefficient values which are greater than the minimum acceptable cut-off value of 0.7 according to Shemwell, Chase, and Schwartz [50]. 
TABle 1: Alpha coefficient for the study's scales.

\begin{tabular}{lccccccc}
\hline & IMTK & IMTA & IMES & EMER & EMIN & EMID & AMOT \\
\hline Alpha & 0.764 & 0.725 & 0.798 & 0.769 & 0.829 & 0.776 & 0.893 \\
\#items & 4 & 4 & 4 & 4 & 4 & 4 & 4 \\
\hline
\end{tabular}

3.2. Preliminarily Analyses. Preliminarily analyses were conducted; no missing values existed; univariate normal distribution was carried out; multivariate normal distribution was examined by using Henze-Zirkler's test $\left(\mathrm{HZ}=3.462269, p_{1} \leq 0.001\right)$, which refers to a violation in the assumption of multivariate normality. The boxplot analysis showed that there were no extreme univariate outliers and no multivariate outliers. The $p$ value of Mahalanobis distance was $p_{1} \leq 0.001$ [51], but it was eliminated from the analysis.

The structural equation model analyses assumed multivariate normality assumption, which is a demand and is practically hard to achieve in many sciences. Therefore, if there is a sufficient sample size and the assumption of multivariate normality is violated, then the maximum likelihood estimation method is robust enough to act against the violation of that assumption, particularly in the case of eliminating or the absence of outliers [52].

Table 2 shows that there were significantly strong positive correlations between intrinsic and extrinsic motivation dimensions which means that intrinsic motivation has a relationship with extrinsic motivation, such that students who had high intrinsic motivation are most likely to have high extrinsic motivation, According to Lemos and Verissimo [53] IM and EM can coexist and are not contradictory. This means that both intrinsic and extrinsic reasons may underlie the student's classroom achievement behaviour while there were insignificant relationships between all intrinsic and extrinsic motivation dimensions and Amotivation, except its relationship with intrinsic motivation to know (IMTK). Means and standard deviations for the seven dimensions are as follows: IMTK: $M=17.35, \mathrm{SD}=2.50$; IMTA $: M=15.92, S D=2.97 ;$ IMES $: M=16.84, \quad \mathrm{SD}=3.04$; EMER $: M=17.15, \mathrm{SD}=2.69 ; \mathrm{EMIN}: M=16.42, \mathrm{SD}=3.39$; EMID $: M=16.85, \quad \mathrm{SD}=2.87 ; \quad$ and $\mathrm{AMOT}: M=9.23$, $\mathrm{SD}=4.82$. These results showed that the study samples relatively had a high degree of intrinsic motivation to know and extrinsic motivation for external regulation, while there was a low degree of Amotivation.

Skewness and kurtosis' coefficients for dimensions were within the absolute value of 2 , which supports the univariate normality [54].

3.3. Principal Component Analysis (PCA). The principal component analysis was conducted to examine the construct of the AMS $(\mathrm{KMO}=0.93>0.6)$ and Bartlett's test of sphericity was $6568.163($ Sig. $=0.00)$ which proves that the data are adequate for PCA according to Tabachnick and Fidell [55].

Then, the Eigenvalues were computed and the analysis showed that there were five Eigenvalues greater than one as shown in Table 3 . Then, parallel analysis was conducted to determine the accurate number of components, whereby its results confirmed the structure of three components.

The analysis was rerun with Equamax rotation, which converged four iterations. The three components explained $56.36 \%$ of the total variance as shown in Table 3 , and which is acceptable as mentioned by Streiner [56].

The items loading computed for the three-factor solution is shown in Table 4.

Table 4 shows that all items loading were significant and greater than 0.32, according to Tabachnick and Fidell [55]. As shown in the table, there exists three domains: extrinsic motivation items (E's), intrinsic motivation items (I's), and Amotivation items (A's). Some items had significant crossloadings (E7, E14, E21, and I23), and the percentage between both loadings was $75 \%$; also, by reviewing the content of these items, it was found that they have somewhat common meanings between intrinsic and extrinsic motivation, which reinforced the idea of discarding these items from the scale. So the items were eliminated as recommended by Samuels et al. [57]. Therefore, the final version of the scale according to PCA was a 24 -item scale with three dimensions.

3.4. Confirmatory Factor Analysis. The CFA was conducted using Amos 23.0 software. The maximum likelihood estimation method was used; the analysis examined three factors with both 28 and 24 items, five factors with 28 items, seven factors with 28 items, and second order model for seven factors with 28 items as well as the best model fit after modifications made. Goodness of fit (GOF) indices were extracted and the criteria were adopted according to many researchers (e.g., $[52,58,59]$, as shown in Table 5.

As shown in Table 5, the CFA results revealed that the three-factor model with 24 items showed the best fit over other models, while the seven-factor model was the worst. Modification indices were checked to improve the fitness of the model, and some modifications were made in light of the logical and theoretical justifications, as shown in Figure 1.

Fit indices after modifications were $\chi^{2}=756.073$; $\chi^{2} / \mathrm{d} f=3.09 ; \mathrm{IFI}=0.903$; CFI $=0.903$ which indicated good fit; $\mathrm{GFI}=0.869$ (indicated adequate fit); RMSEA $=0.072$ (indicated good fit). All items loaded significantly on latent variables (dimensions) as shown in Table 6.

Regression weights were significant at $p=0.001$ for all items and greater than 0.3 , which was considered as an acceptable cut-off point $[60,61]$. Composite reliability was also calculated according to the equation mentioned in Raycov's [62] study. CR was recommended to be above 0.7 , which indicates that items constantly measure the same structure [63].

Corrected item total correlations for each item were computed; all items correlations were greater than 0.3 , which indicates that the corresponding item correlates well with the overall scale $[64,65]$, as well as Cronbach's alpha coefficient for the three dimensions computed, and their values' indicated a very good reliability. 
TABle 2: Descriptive statistics.

\begin{tabular}{|c|c|c|c|c|c|c|c|c|c|c|}
\hline Scale & IMTK & IMTA & IMES & EMER & EMIN & EMID & Mean & $\mathrm{SD}$ & Skew. & Kur. \\
\hline IMTK & - & & & & & & 17.3466 & 2.50141 & -0.788 & 0.156 \\
\hline IMTA & $0.695^{* *}$ & - & & & & & 15.9202 & 2.96877 & -0.642 & 0.106 \\
\hline IMES & $0.757^{* *}$ & $0.725^{* *}$ & - & & & & 16.8354 & 3.04020 & -0.843 & 0.131 \\
\hline EMER & $0.669^{* *}$ & $0.540^{* *}$ & $0.666^{* *}$ & - & & & 17.1546 & 2.68813 & -0.783 & -0.032 \\
\hline EMIN & $0.614^{* *}$ & $0.587^{* *}$ & $0.771^{* *}$ & $0.670^{* *}$ & - & & 16.4165 & 3.38801 & -1.080 & 1.154 \\
\hline EMID & $0.504^{* *}$ & $0.388^{*}$ & $0.485^{* *}$ & $0.738^{* *}$ & $0.596^{* *}$ & - & 16.8479 & 2.86780 & -0.672 & -0.145 \\
\hline AMOT & $-0.176^{* *}$ & 0.078 & -0.066 & -0.073 & 0.003 & 0.044 & 9.2269 & 4.82140 & 0.612 & -0.746 \\
\hline
\end{tabular}

TABLE 3: Eigenvalues for AMS.

\begin{tabular}{lcccccc}
\hline Component & Eigenvalue & \% variance & Cumulative \% & Rotated eigenvalue & Rotated \% variance & Rotated cumulative \% \\
\hline 1 & 10.487 & 37.453 & 37.453 & 6.448 & 23.028 & 23.028 \\
2 & 3.397 & 12.131 & 49.584 & 5.894 & 21.050 & 12.277 \\
3 & 1.896 & 6.772 & 56.356 & 3.438 & & 56.356 \\
4 & 1.160 & 4.144 & 60.500 & & & \\
5 & 1.079 & 3.855 & 64.355 & & & \\
\hline
\end{tabular}

TABLE 4: AMS items loading.

\begin{tabular}{|c|c|c|c|}
\hline $\begin{array}{l}\text { Item } \\
\text { number }\end{array}$ & $\begin{array}{c}\text { Intrinsic } \\
\text { motivation }\end{array}$ & $\begin{array}{c}\text { Extrinsic } \\
\text { motivation }\end{array}$ & Amotivation \\
\hline E1 & & 0.661 & \\
\hline $\mathrm{I} 2$ & 0.511 & & \\
\hline E3 & & 0.526 & \\
\hline $\mathrm{I} 4$ & 0.652 & & \\
\hline A5 & & & 0.849 \\
\hline I6 & 0.706 & & \\
\hline E7 & 0.551 & 0.456 & \\
\hline E8 & & 0.788 & \\
\hline I9 & 0.691 & & \\
\hline E10 & & 0.679 & \\
\hline I11 & 0.599 & & \\
\hline A12 & & & 0.785 \\
\hline I13 & 0.661 & 0.360 & \\
\hline E14 & 0.428 & 0.570 & \\
\hline E15 & & 0.709 & \\
\hline I16 & 0.639 & 0.405 & \\
\hline E17 & & 0.713 & \\
\hline I18 & 0.666 & & \\
\hline A19 & & & 0.889 \\
\hline $\mathrm{I} 20$ & 0.622 & & \\
\hline $\mathrm{E} 21$ & 0.464 & 0.496 & \\
\hline E22 & & 0.795 & \\
\hline I23 & 0.438 & 0.487 & \\
\hline E24 & & 0.586 & \\
\hline $\mathrm{I} 25$ & 0.663 & & \\
\hline A26 & & & 0.890 \\
\hline I27 & 0.664 & & \\
\hline
\end{tabular}

\section{Discussion}

The current study aimed at verifying the construct validity of the AMS in the Arab environment using PCA and CFA. The results suggested that the AMS has adequate reliability. Cronbach's alpha values for the seven dimensions ranged between 0.725 and 0.893 , which is consistent with the values of the original version of the AMS [34].
Factor analysis results did not support the seven-scale factors, as the scale items were loaded on three dimensions: intrinsic motivation, extrinsic motivation, and Amotivation. This result is online with the SDT, which indicated that motivation consists of three general dimensions located on a continuum of the upper end of intrinsic motivation, then extrinsic motivation, and finally Amotivation [8].

The current study agrees with the study of Natalya and Purwanto [46] that was conducted in Indonesia and concluded that the items of the academic motivation scale were loaded on three dimensions: intrinsic motivation, extrinsic motivation, and Amotivation. The results partially agreed with those of several studies that did not confirm the sevenfactor model, such as that of Taghipour Ali Hosein et al. [39] which concluded that the scale consists of two dimensions, and the study by Alruaili [66] concluded that the scale consists of four dimensions. Some studies [41], Lim and Chapman (2014) [67], concluded that the scale consists of five dimensions. Abu Awad's (2009) study concluded that the scale consists of six dimensions, while the results differed with the findings of Vallerand et al. [34]; which indicated that the factorial structure of the scale consists of seven dimensions.

This difference in determining the factorial structure of the AMS may be due to multiple factors, including the cultural factors between the Arab environment (in which the current study was conducted) and the Western environments, including the French environment in which the original study of the AMS was conducted and the Canadian environment in which the factorial structure of the AMS was verified in the English version by Vallerand et al. [34]. This result supports the idea of cultural differences in the students' academic motivation and is consistent with the idea of Allport [26] that motivations are not general or common to all individuals, as they differ in different civilisations (cultures).

Allport [26] mentioned that there are no common motives to all human beings, for every individual is a fabric 
TABLE 5: GOF indices for the study scales.

\begin{tabular}{|c|c|c|c|c|c|c|c|c|c|}
\hline Model & $\chi^{2}$ & $\chi^{2} / \mathrm{d} f$ & IFI & CFI & GFI & RMSEA & $\begin{array}{l}\text { \# sample } \\
\text { moments }\end{array}$ & \# parameters & $D f$ \\
\hline Recommended value & Less the better & $\chi^{2} / \mathrm{d} f \leq 5$ & $\geq 0.90$ & $\geq 0.90$ & $\geq 0.90$ & RMSEA $\leq 0.08$ & & & \\
\hline Three factors with 28 items & $1736.025^{* *}$ & 4.960 & 0.783 & 0.782 & 0.770 & 0.099 & 406 & 56 & 350 \\
\hline Three factors with 24 items & $1244.966^{* *}$ & 4.940 & 0.8120 & 0.8110 & 0.8160 & 0.0990 & 300 & 48 & 252 \\
\hline Five factors with 28 items & $2242.247^{* *}$ & 6.406 & 0.704 & 0.703 & 0.711 & 0.116 & 406 & 56 & 350 \\
\hline Seven factors with 28 items & $3034.794^{* *}$ & 8.671 & 0.5800 & 0.5780 & 0.5860 & 0.1380 & 406 & 56 & 350 \\
\hline $\begin{array}{l}\text { Second order seven factors with } 28 \\
\text { items }\end{array}$ & $2618.741^{* *}$ & 7.842 & 0.645 & 0.622 & 0.622 & 0.127 & 406 & 56 & 350 \\
\hline Modified three factors with 24 items & $756.073^{* *}$ & 3.09 & 0.903 & 0.903 & 0.869 & 0.072 & 300 & 56 & 244 \\
\hline
\end{tabular}

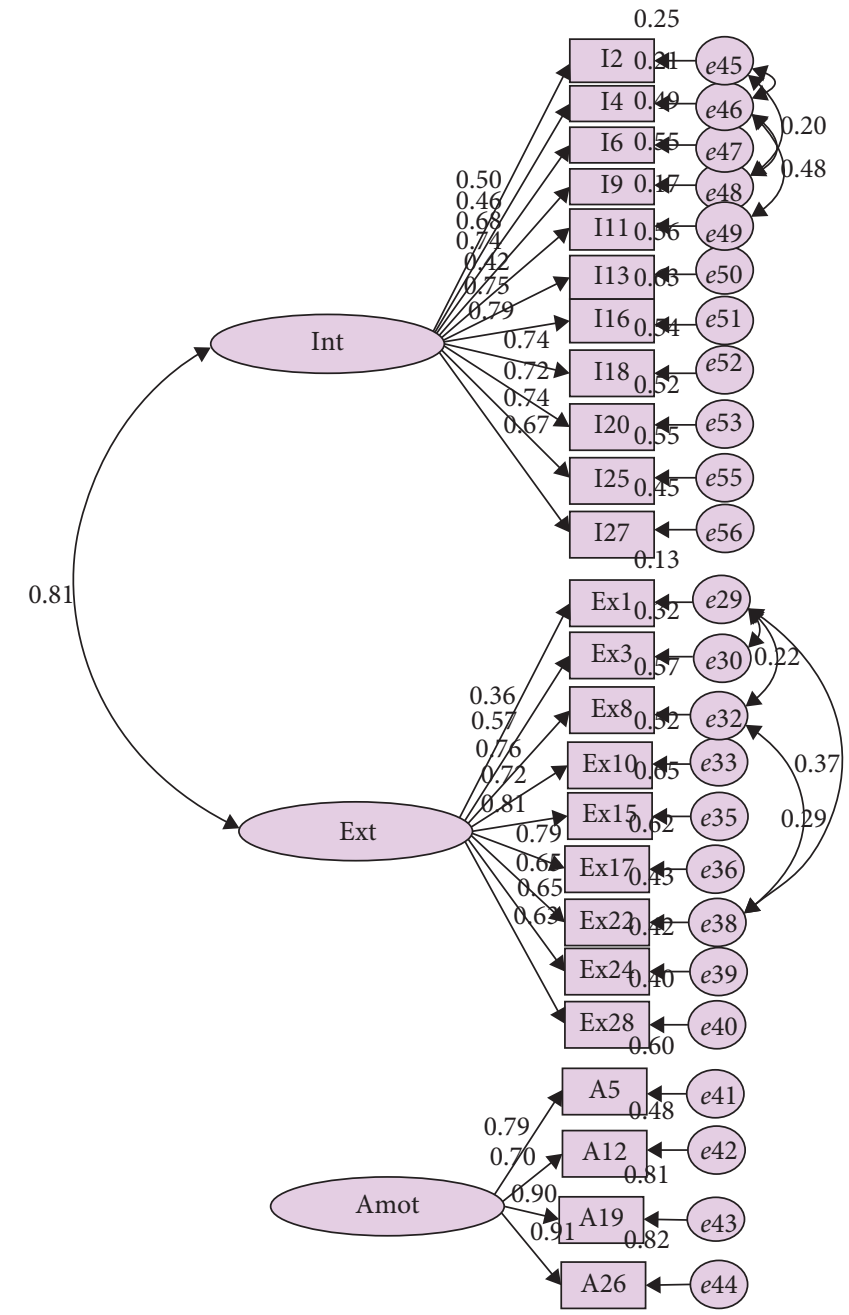

Figure 1: Modifications made in light of the logical and theoretical justifications.

alone, just as every civilisation is a fabric alone. The SDT considers that human behaviour is formed through the interaction between an individual and his social environment, which affects the motivation of individuals due to psychological and natural needs that they try to satisfy, regardless of the social environment they belong to [19, 29, 48]. Fairchild et al. [38] observed that intrinsic motivation and extrinsic motivation are not two exclusive constructs, but rather a continuum. It is, therefore, probable that the grouping of three dimensions can still be accepted as the best grouping for the AMS at the current study.

Also, this discrepancy in the results of studies that dealt with the factorial structure of the AMS confirms the necessity of conducting more studies on samples from different cultures in order to find out the truth about the factorial structure of the AMS and to verify its psychometric properties and also to answer the question: "Does the factorial structure of the AMS differ across cultures?" In other words, 
TABLE 6: Items loaded on latent variables (dimensions).

\begin{tabular}{|c|c|c|c|c|c|}
\hline \multicolumn{2}{|r|}{ Extrinsic } & \multicolumn{2}{|r|}{ Intrinsic } & \multicolumn{2}{|r|}{ Amotivation } \\
\hline \# & Standardised coef. & $\#$ & Standardised coef. & \# & Standardised coef. \\
\hline Ex1 & $0.361^{* * *}$ & $\operatorname{In} 2$ & $0.498^{* * *}$ & A5 & $0.793^{* * *}$ \\
\hline Ex3 & $0.566^{* * *}$ & In 4 & $0.462^{* * *}$ & A12 & $0.696^{* * *}$ \\
\hline Ex8 & $0.757^{* * *}$ & In6 & $0.694^{* * *}$ & A19 & $0.898^{* * *}$ \\
\hline Ex10 & $0.719^{* * *}$ & In9 & $0.740^{* * *}$ & A26 & $0.906^{* * *}$ \\
\hline Ex15 & $0.807^{* * *}$ & In11 & $0.417^{* * *}$ & & \\
\hline Ex17 & $0.786^{* * *}$ & $\operatorname{In} 13$ & $0.748^{* * *}$ & & \\
\hline Ex22 & $0.652^{* * *}$ & In16 & $0.791^{* * *}$ & & \\
\hline Ex24 & $0.651^{* * *}$ & $\operatorname{In} 18$ & $0.735^{* * *}$ & & \\
\hline \multirow[t]{3}{*}{ Ex28 } & $0.629^{* * *}$ & $\operatorname{In} 20$ & $0.721^{* * *}$ & & \\
\hline & & $\operatorname{In} 25$ & $0.743^{* * *}$ & & \\
\hline & & $\operatorname{In} 27$ & $0.668^{* * *}$ & & \\
\hline CR & 0.877 & $\mathrm{CR}$ & 0.895 & CR & 0.896 \\
\hline$\#$ & Corrected item-total correlation & \# & Corrected item-total correlation & \# & Corrected item-total correlation \\
\hline Ex1 & 0.454 & $\operatorname{In} 2$ & 0.483 & A5 & 0.754 \\
\hline Ex3 & 0.537 & In 4 & 0.531 & A12 & 0.670 \\
\hline Ex8 & 0.742 & In6 & 0.672 & A19 & 0.816 \\
\hline Ex10 & 0.660 & In9 & 0.701 & A26 & 0.823 \\
\hline Ex15 & 0.714 & In11 & 0.463 & & \\
\hline Ex17 & 0.713 & $\operatorname{In} 13$ & 0.681 & & \\
\hline Ex22 & 0.694 & In 16 & 0.696 & & \\
\hline Ex24 & 0.587 & In 18 & 0.676 & & \\
\hline \multirow[t]{3}{*}{ Ex28 } & 0.518 & $\operatorname{In} 20$ & 0.663 & & \\
\hline & & $\operatorname{In} 25$ & 0.686 & & \\
\hline & & $\operatorname{In} 27$ & 0.628 & & \\
\hline Alpha & 0.871 & Alpha & 0.892 & Alpha & 0.893 \\
\hline
\end{tabular}

${ }^{* * *} p<0.001$.

"Do human motives differ according to culture?" This may contribute to confirming or denying that motivation is influenced by cultural factors.

The AMS has been used in different cultures with different models (factors) and different languages. This difference in languages and cultures may lead to real problems in language and expression, and this in turn may affect the structure of the scale and its psychometric properties. The use of the scale in the Arabic language on university students in Jordan demonstrates the need to do more to develop a special version of the AMS that is compatible with the Arabic culture, as well as the Arabic language.

The findings of the current study need to be supported by other similar studies that agree or disagree with it and to conduct other studies on different samples of age, culture, place of residence, and other demographic variables that may also help in identifying the reality of the factorial structure of the AMS.

Finally, such results may be a clear invitation to researchers and those interested in studying academic motivation to choose measures that fit the culture in which the study is conducted. For example, in eastern cultures, success is driven by fear of failure and what follows it in terms of a social view of failure (external motivation), while western cultures promote success in a desire for success and not fear of failure (internal motivation). From this perspective, it is important to choose a scale that is culturally unbiased.

\section{Conclusion}

From the results of the current study and its discussion, we can conclude that the factorial structure of the AMS differs from one culture to another, which places limitations in its use. This conclusion was suggested because of the results of the studies conducted in different environments about the structure of the AMS. If this conclusion is correct, then it will reinforce the idea that human motivation is affected by cultural factors, and this requires work to provide measures of motivation which are free of cultural factors-what is appropriate in one culture may not be appropriate in another. Hence, the researcher recommends conducting more studies in different environments on a variety of samples in order to provide measures of motivation that have psychometric properties and a good factor structure because of the great importance of academic motivation in students orientations towards learning and success.

\section{Data Availability}

No data were used to support this study.

\section{Conflicts of Interest}

The author declares that he has no conflicts of interest. 


\section{Acknowledgments}

The author thanks all the participants who took part in this study and enabled this research to be possible.

\section{References}

[1] S. Abu Jadu, Educational Psychology, Al Masirah House, Amman, Jordan, 1998.

[2] J. W. Atkinson, An Introduction to Motivation, Van Nostrand, Princeton, NJ, USA, 1964.

[3] M. Mustafa, "Studying the academic motivation among education college students in Al-Arish," Journal of Psychological and Educational Research, vol. 2, pp. 149-177, 1998.

[4] D. H. Schunk, "Introduction to the special section on motivation and efficacy," Journal of Educational Psychology, vol. 82, no. 1, pp. 3-6, 1990.

[5] L. M. Berry, Psychology at Work, McGraw-Hill, Singapore, 2nd edition, 1998.

[6] G. Moorhead and R. W. Griffin, Organizational Behavior Managing People \& Organization, Houghton Mifflin company, Boston, MA, USA, 5th edition, 1998.

[7] A. Bleakley, "Learning as an aesthetic practice: motivation through beauty in higher education," in Motivating Students, S. Brown, S. Armstrong, and G. Thompson, Eds., pp. 165-172, Kogan Page, London, UK, 1998.

[8] R. M. Ryan and E. L. Deci, "Self-determination theory and the facilitation of intrinsic motivation, social development, and well-being," American Psychologist, vol. 55, no. 1, pp. 68-78, 2000.

[9] A. Lorsi, The Reference in the Circular, Josoor for Publication, Algeria, 2014.

[10] M. Abdel Qader and A. Al-Quraiti, Principles of Psychology, Egyptian Nahda Library, Cairo, Egypt, 1997.

[11] R. Hamitton and E. Ghatala, Learning and Instruction, McGraw-Hill, Inc, New York, NY, USA, 1994.

[12] R. J. Cocks and H. M. G. Watt, "Self- perceptions of academic competence: how they develop and how they relate to intrinsic motivation for learning in English and Math for year six students," in Proceedings of the AARE Annual Conference, Fremantle Perth, Australia, December 2001.

[13] P. R. Pintrich and E. V. De Groot, "Motivational and selfregulated learning components of classroom academic performance," Journal of Educational Psychology, vol. 82, no. 1, pp. 33-40, 1990.

[14] T. J. Newby, "Classroom motivation: strategies of first-year teachers," Journal of Educational Psychology, vol. 83, no. 2, pp. 195-200, 1991.

[15] C. S. Dweck and E. L. Leggett, "A socialcognitive approach to motivation and personality," Psychological Review, vol. 95, no. 2, pp. 256-273, 1988.

[16] P. Race, "Teaching: creating a thirst for learning," in Motivating Students, S. Brown, S. Armstrong, and G. Thompson, Eds., pp. 47-58, Kogan Page, London, UK, 1998.

[17] J. Brophy, Motivating Student to Learn, McGraw-Hill companies, Inc, New York, NY, USA, 1999.

[18] J. Gnoth and B. Juric, "Students' Motivation to Study Introductory Marketing," Educational Psychology, vol. 16, no. 4, pp. 389-405, 1996.

[19] E. Deci and R. Ryan, Intrinsic Motivation and the Self-Determination of Human Behavior, Plenum Press, New York, NY, USA, 1985.

[20] R. M. Baron and D. A. Kenny, "The moderator-mediator variable distinction in social psychological research: conceptual, strategic, and statistical considerations," Journal of Personality and Social Psychology, vol. 51, no. 6, pp. 1173-1182, 1986.

[21] A. E. Gottfried, J. S. Fleming, and A. W. Gottfried, "Role of parental motivational practices in children's academic intrinsic motivation and achievement," Journal of Educational Psychology, vol. 86, no. 1, pp. 104-113, 1994.

[22] F. Cury, S. Biddle, J. P. Famose, P. Sarrazin, M. Durand, and M. Goudas, "Personal and situational factors influencing intrinsic interest of adolescent girls in school physical education: a structural equation modelling analysis," Educational Psychology, vol. 16, no. 3, pp. 305-315, 1996.

[23] A. E. Gottfried, "Academic intrinsic motivation in elementary and junior high school students," Journal of Educational Psychology, vol. 77, no. 6, pp. 631-645, 1985.

[24] A. K. Boggiano and M. Barrett, "Performance and motivational deficits of helplessness: the role of motivational orientations," Journal of Personality and Social Psychology, vol. 49, no. 6, pp. 1753-1761, 1985.

[25] N. Azid, "Predicting the academic achievement of the classroom actions and emotions of fifth grade primary students," in Proceeding of the Educational Research Conference in the Arab World, Where To?, Human Resources Development Center, Amman, Jordan, December 1998.

[26] G. W. Allport, "Personality: a problem for science or a problem for art?” Revista de Psihologie, vol. 1, pp. 488-502, 1938.

[27] L. Fateem, Contemporary Theories of Learning, Nahda Library, Cairo, Egypt, 2nd edition, 1996.

[28] A. Nashwati, Educational Psychology, Dar Al-Furqan, Amman, Jordan, 5th edition, 1991.

[29] E. Deci and R. Ryan, Intrinsic Motivation and Self-Determination in Human Behavior, Plenum Press, New York, NY, USA, 1990.

[30] D. McClelland, Human Motivation, Scott freeman, Glenview, IL, USA, 1985.

[31] J. W. Atkinson and T. Feather, A Theory of Achievement Motivation, Wiley, New York, NY, USA, 1986.

[32] M. R. Lepper, "Intrinsic and Extrinsic Motivational Orientations in the Classroom: Age Differences and Academic Correlates," Journal of Educational Psychology, vol. 97, no. 2, pp. 184-196, 2005.

[33] R. Bhat and A. Naik, "Relationship of academic intrinsic motivation and psychological well-being among students," International Journal of Modern Social Sciences, vol. 5, no. 1, pp. 66-74, 2016.

[34] R. J. Vallerand, L. G. Pelletier, M. R. Blais, N. M. Briere, C. Senecal, and E. F. Vallieres, "The academic motivation scale: a measure of intrinsic, extrinsic, and a motivation in education," Educational and Psychological Measurement, vol. 52, no. 4, pp. 1003-1017, 1992.

[35] J. Stover, G. de la Iglesia, A. Rial, and M. F. Fernández Liporace, "Academic motivation scale: adaptation and psychometric analyses for high school and college students," Psychology Research and Behavior Management, vol. 5, pp. 71-83, 2012.

[36] K. O. Cokley, "Examining the validity of the academic motivation scale by comparing scale construction to self-determination theory," Psychological Reports, vol. 86, no. 2, pp. 560-564, 2000.

[37] K. O. Cokley, N. Bernard, D. Cunningham, and J. Motoike, "A psychometric investigation of the academic motivation scale using a United States sample," Measurement and Evaluation 
in Counseling and Development, vol. 34, no. 2, pp. 109-119, 2001.

[38] A. J. Fairchild, S. J. Horst, S. J. Finney, and K. E. Barron, "Evaluating existing and new validity evidence for the academic motivation scale," Contemporary Educational Psychology, vol. 30, no. 3, pp. 331-358, 2005.

[39] F. EL-yazidi and F. Louzani, "The factorial structure of the academic motivation scale," Educational and Psychological Sciences Journal, vol. 4, no. 1, pp. 149-173, 2017.

[40] A. D. Ochoco, A Psychometric Validation of Academic Motivation Scale (AMS) in the Filipino Context, Adventist College, Manila, Philippines, 2007.

[41] F. Alivernini and F. Lucidi, "The academic motivation scale (AMS): factorial structure, invariance, and validity in the Italian context," TMP, vol. 15, no. 4, pp. 211-220, 2008.

[42] M. H. U. Karaguven, "The adaptation of academic motivation scale to Turkish," Educational Sciences: Theory \& Practice, vol. 12, no. 4, pp. 2611-2618, 2012.

[43] S. Y. Lim and E. Chapman, "Adapting the academic motivation scale for use in pre-tertiary mathematics classrooms," Mathematics Education Research Journal, vol. 27, no. 3, pp. 331-357, 2014.

[44] I. S. Caleon, M. G. L. Wui, J. P.-L. Tan, C. L. Chiam, T. C. Soon, and R. B. King, "Cross-cultural validation of the academic motivation scale: a Singapore investigation," Child Indicators Research, vol. 8, no. 4, pp. 925-942, 2015.

[45] B. Zhang, Y. M. Li, J. Li, Y. Li, and H. Zhang, "The revision and validation of the academic motivation scale in China," Journal of Psychoeducational Assessment, vol. 34, no. 1, pp. 15-27, 2016.

[46] L. Natalya and C. V. Purwanto, "Exploratory and confirmatory factor analysis of the academic motivation scale (AMS)-Bahasa Indonesia," Makara Human Behavior Studies in Asia, vol. 22, no. 1, pp. 29-42, 2018.

[47] F. Abu Awad, "The factorial structure of the academic motivation scale (AMS): a psychometric study on a sample of sixth and tenth grades students in UNRWA schools in Jordan," Damascus University Journal, vol. 25, no. 3-4, pp. 433-471, 2009.

[48] E. L. Deci and R. M. Ryan, "A motivational approach to self: integration in personality," in Nebraska Symposium on Motivation: Perspectives on Motivation, R. A. Dienstbier, Ed., pp. 237-288, University of Nebraska Press, Lincoln, NE, USA, 1991, https://europepmc.org/article/med/2130258.

[49] S. A. S. Algharaibeh, "Should I ask for help? the role of motivation and help-seeking in students' academic achievement: a path analysis model," Cypriot Journal of Educational Sciences, vol. 15, no. 5, pp. 1128-1145, 2020.

[50] J. T. Shemwell, C. C. Chase, and D. L. Schwartz, "Seeking the general explanation: a test of inductive activities for learning and transfer," Journal of Research in Science Teaching, vol. 52, no. 1, pp. 58-83, 2015.

[51] R. Momani and S. Gharaibeh, "Investigating the construct validity of Sternberg's triarchic abilities test level-H (Arabic version)," International Journal of Advanced and Applied Sciences, vol. 4, no. 11, pp. 28-34, 2017.

[52] P. M. Bentler and C.-P. Chou, "Practical issues in structural modeling," Sociological Methods \& Research, vol. 16, no. 1, pp. 78-117, 1987.

[53] M. S. Lemos and L. Veríssimo, "The relationships between intrinsic motivation, extrinsic motivation, and achievement, along elementary school," Procedia-Social and Behavioral Sciences, vol. 112, pp. 930-938, 2014.
[54] D. George and M. Mallery, SPSS for Windows Step by Step: A Simple Guide and Reference, Pearson, Boston, MA, USA, 10th edition, 2010.

[55] B. G. Tabachnick and L. S. Fidell, Using Multivariate Statistics, Pearson, Harlow, UK, 6th edition, 2014.

[56] D. L. Streiner, "Figuring out factors: the use and misuse of factor analysis," The Canadian Journal of Psychiatry, vol. 39, no. 3, pp. 135-140, 1994.

[57] P. Samuels, J. Hall, B. Montz, J. Schanze, S. Simonovic, and K. Takara, "Looking back and looking forward," Journal of Flood Risk Management, vol. 10, no. 1, pp. 3-4, 2017.

[58] K. A. Bollen and R. Stine, "Direct and indirect effects: classical and bootstrap estimates of variability," Sociological Methodology, vol. 20, pp. 115-140, 1990.

[59] L. R. Fabrigar, D. T. Wegener, R. C. MacCallum, and E. J. Strahan, "Evaluating the use of exploratory factor analysis in psychological research," Psychological Methods, vol. 4, no. 3, pp. 272-299, 1999.

[60] S. P. Lee, C. Ong, V. Sagayadevan et al., "Hoarding symptoms among psychiatric outpatients: confirmatory factor analysis and psychometric properties of the Saving Inventory-revised (SI-R)," BMC Psychiatry, vol. 16, no. 1, p. 364, 2016.

[61] M. Yusoff, A. Abdul Rahim, R. Abd Aziz et al., "The validity and reliability of the USM personality inventory (USMaP-i): its use to identify personality of future medical students," International Medical Journal, vol. 18, no. 4, pp. 283-290, 2011.

[62] T. Raykov, "Estimation of composite reliability for congeneric measures," Applied Psychological Measurement, vol. 21, no. 2, pp. 173-184, 1997.

[63] J. Hair, W. Black, B. Babin, R. Anderson, and R. Tatham, Multivariate Data Analysis, Pearson International Edition, New Jersey, NJ. USA, 2006.

[64] B. S. Everitt, The Cambridge Directory of Statistics, CPU, 2nd edition, 2002.

[65] A. Field, Discovering Statistics Using SPSS, Sage, London, UK, 2nd edition, 2005.

[66] W. Alruaili, "Reliability of an Arabic version of the academic motivation scale (AMS) on students at Al-Jouf university," Doctoral dissertation, Graduate School of Wayne State University, Detroit, Michigan, 2020.

[67] T. Manger, J. Hetland, L. Ø. Jones, O. J. Eikeland, and A. E. Asbjørnsen, "Prisoners' academic motivation, viewed from the perspective of self-determination theory: evidence from a population of Norwegian prisoners," International Review of Education, vol. 66, no. 4, pp. 551-574, 2020. 